\title{
RÉGIMEN TÉRMICO DE SUELOS DEL MACIZO CENTRAL DE PICOS DE EUROPA (ESPAÑA)
}

\section{Ground thermal regime in Central Massif of Picos de Europa (Spain)}

\author{
Alfonso Pisabarro Pérez ${ }^{1, *}$, Enrique Serrano Cañadas ${ }^{1}$ y Juan José González Trueba² \\ ${ }^{1}$ Dpto. de Geografía, Universidad de Valladolid, P. ${ }^{\circ}$ Prado Magdalena. 47011 Valladolid. alfonso.pisabarro@uva.es \\ ${ }^{1}$ Dpto. de Geografía, Universidad de Valladolid, P. ${ }^{\circ}$ Prado Magdalena. 47011 Valladolid. serranoe@fyl.uva.es \\ ${ }^{2}$ Dpto. de Geografía. Centro Universitario CIESE. Fundación Comillas. Avda. de la Universidad Pontificia, s/n. 39540 Santander. \\ gonzalezj@fundacioncomillas.es \\ *Autor corresponsal
}

Recibido: 23-01-2015. Aceptado: 19-03-2015. Fecha de publicación on-line: 10-09-2015

Citation / Cómo citar este artículo: Pisabarro Pérez, A., Serrano Cañadas, E. y González Trueba, J.J. (2015). Régimen térmico de suelos del macizo central de Picos de Europa (España). Pirineos, 170, e010. doi: http://dx.doi.org/10.3989/Pirineos.2015.170003

RESUMEN: El estudio de las temperaturas del suelo en la alta montaña húmeda templada tiene un gran interés porque determina gran parte de los procesos geomorfológicos en un ámbito periglaciar. El objetivo de la investigación es analizar los principales procesos térmicos del suelo a lo largo del año en diferentes altitudes y emplazamientos en el macizo central de Picos de Europa (43⒈ $\left.{ }^{\prime}-43^{\circ} 7^{\prime} \mathrm{N} ; 5^{\circ} 7^{\prime}-4^{\circ} 36^{\prime} \mathrm{O}\right)$. La metodología ha sido ya aplicada en los Alpes y ha consistido en la obtención de temperaturas a través de 12 microsensores térmicos enterrados a $10 \mathrm{~cm}$ con emplazamientos muy diversos entre los 1.110 y 2.535 m.s.n.m. distribuidos buscando el escalonamiento altitudinal y los emplazamientos más fríos. En el artículo se presentan los siguientes parámetros: régimen térmico, ciclos de hielo y deshielo, índice de helada, duración y profundidad de la helada. El régimen térmico varía de acuerdo a las condiciones topoclimáticas de cada emplazamiento aunque se han podido estimar cuatro fases anuales en función del comportamiento del manto nival que se induce de los registros térmicos. El período del año mejor analizado ha sido el invierno y la primavera, cuando el suelo se encuentra protegido por un grueso manto nival y, en consecuencia, los ciclos de hielo y deshielo se anulan. Durante este período los registros nos han permitido afirmar la existencia de suelos helados estacionales en cinco emplazamientos y la posibilidad en otros seis. Sin embargo, no han sido suficientes para encontrar evidencias de permafrost.

PALABRAS CLAVE: Régimen térmico de suelos; alta montaña templada; manto nival; geomorfología.

ABSTRACT: The study of ground temperatures in a wet and temperate high mountain has got large interest because determines significant geomorphological processes in a periglacial environment. The aim of the research is to analyse the main thermal processes of ground along the year at different altitudes in the central massif of Picos de Europa $\left(43^{\circ} 18^{\prime}-43^{\circ} 7^{\prime} \mathrm{N} ; 5^{\circ} 7^{\prime}-4^{\circ} 36^{\prime} \mathrm{W}\right)$. Methodology has been used in the Alps and consists in temperature data that were obtained by 12 temperature data loggers undertaken at $10 \mathrm{~cm}$ with diverse locations between 1110 and $2535 \mathrm{~m}$ a.s.l. searching for altitudinal stepping and the coldest locations. The paper show the next parameters: thermal regime, freeze and thaw cycles, freeze index, duration and depth freeze. The thermal regime varies according topocli- 


\section{• A. PISABARRO PÉREZ, E. SERRANO CAÑADAS Y J.J. GONZÁLEZ TRUEBA}

matic conditions but it was possible to determinate four annual phases in function of snow cover behavior induced through the thermal registers. The year period best analyzed has been winter and spring, when the ground is protected by a big snow cover and in consequence there are not freeze and thaw cycles. During this period registers allow to asseverate the evidence of seasonal freeze grounds in five locations and the possibility in another six. However the data were not enough to discover permafrost.

KEY WORDS: Ground Thermal Regime; temperate high mountain; snow cover; geomorphology.

\section{Introducción}

El estudio de las temperaturas de los suelos en ambientes periglaciares de montaña tiene un gran interés ya que la temperatura de los mismos determina buena parte de los procesos geomorfológicos en la alta montaña. Es importante conocer su comportamiento a lo largo del año para determinar los umbrales críticos que desencadenan estos procesos.

En el contexto internacional, el estudio térmico de los suelos en la alta montaña se ha desarrollado desde los años 70, en la mayoría de los casos buscando la existencia de permafrost en ámbitos muy diferentes a los de la Cordillera Cantábrica y con objetivos muy distintos. La cordillera alpina ha sido el lugar donde más estudios se han realizado, sobretodo, acerca del permafrost de montaña. Por su importancia en el estudio de las bases físicas y el establecimiento de umbrales térmicos para la presencia de permafrost de montaña y suelos helados estacionales destacan los trabajos de Haeberli (1973), Washburn (1979) y French (2007). Paralelamente, el estudio de las implicaciones térmicas del manto nival ha tenido un gran desarrollo como evidencia Zhang (2005) y sobretodo el estudio específico del régimen térmico por Ishikawa (2003) y Delaloye (2004). Es precisamente este último trabajo del que se toma la metodología para realizar el presente análisis de Picos de Europa. En la década actual los regímenes térmicos de suelos están siendo ampliamente estudiados, si bien, se centran prioritariamente en medios de alta montaña con permafrost y en su degradación, muy diferenciados de la alta montaña templada atlántica.

En otras montañas de la Península Ibérica se han realizado trabajos que relacionan los procesos térmicos con los geomorfológicos, como el realizado en la Serra da Estrela (Vieira et al., 2003). En Sierra Nevada se pudieron determinar ciclos térmicos de hielo y deshielo en diferentes lugares de manto nival inestable (Salvador et al., 2012) y en la Sierra de Guadarrama, Andrés y Palacios (2010) realizaron la monitorización de las temperaturas del aire y del suelo sin encontrar una importante penetración de la helada. En los Pirineos, los regímenes térmicos de suelos se han estudiado en relación al permafrost y los suelos helados estacionales (Serrano et al., 2000; Lugon et al. 2005; Julián y Chueca, 2007; González González, 2014).

En la Cordillera Cantábrica hay pocas investigaciones relativas al comportamiento térmico del suelo. Destaca el estudio del régimen térmico en glaciares rocosos en la cuenca del Alto Sil (Santos González et al., 2009;
Santos González, 2011) en altitudes muy similares y que ha servido para comparar los resultados obtenidos. En el macizo de Fuentes Carrionas, Pellitero (2013) realizó el análisis térmico de suelos en el cual obtuvo índices de helada, ciclos de hielo y deshielo, días por debajo de $0^{\circ} \mathrm{C}$, y las diferentes fases térmicas para cada estación del año.

El lugar seleccionado para el análisis es el Macizo Central de los Picos de Europa (Figura 1), el macizo con las mayores altitudes de la Cordillera Cantábrica (2.648 m.s.n.m.). Es una montaña bien desarrollada con una superficie de $150 \mathrm{~km}^{2}$ individualizada mediante profundos desfiladeros y compartida por León, Asturias y Cantabria. El relieve es muy abrupto y se compone de grandes torres y profundos «jous» que individualizan «grupos de cumbres». La litología es calcárea con espesores de caliza superiores a los 2.000 m.s.n.m., aunque está diferenciada en tres facies. Las precipitaciones son intensas, gran parte en forma de nieve, y superan los $1.900 \mathrm{~mm}$ anuales (González Trueba y Serrano, 2010) debido a la cercanía del mar. La nieve caracteriza un espacio en el que aún se puede encontrar hielo fósil. Esta puede caer incluso en verano en algún punto, pero normalmente las nevadas importantes llegan con los frentes otoñales y desencadenan intensos procesos kársticos superficiales y subterráneos dirigidos por la fracturación y la estratificación. La nieve, además, combina su acción con fenómenos térmicos como la gelifracción sobre formaciones heredadas de origen glaciar y periglaciar, y sobre la roca desnuda dominante por encima de los 1.800 m.s.n.m. Este ámbito ha sido denominado como subpiso crionival (González Trueba, 2007).

El citado emplazamiento es, por lo tanto, un espacio muy adecuado para la investigación de los límites de los procesos periglaciares así como para la búsqueda de respuestas a diferentes formas de origen térmico como los suelos ordenados, los montículos de hielo, los flujos de derrubios o los lóbulos de gelifluxión. Sin embargo, aunque conocemos la existencia de estas formas, no conocemos en profundidad los procesos térmicos que las originan.

Por esta razón, la investigación tiene como objetivo general el estudio del comportamiento térmico de los suelos en un macizo en el que no existen estudios relevantes en profundidad, sino tan solo partes y fragmentos de trabajos. El estudio térmico de los suelos en el Macizo Central de los Picos de Europa fue por primera vez tratado parcialmente por Castañón y Frochoso (1998). Obtuvieron registros de temperaturas en las paredes desprovistas de manto nival del Jou de Cabrones. En esta investiga- 


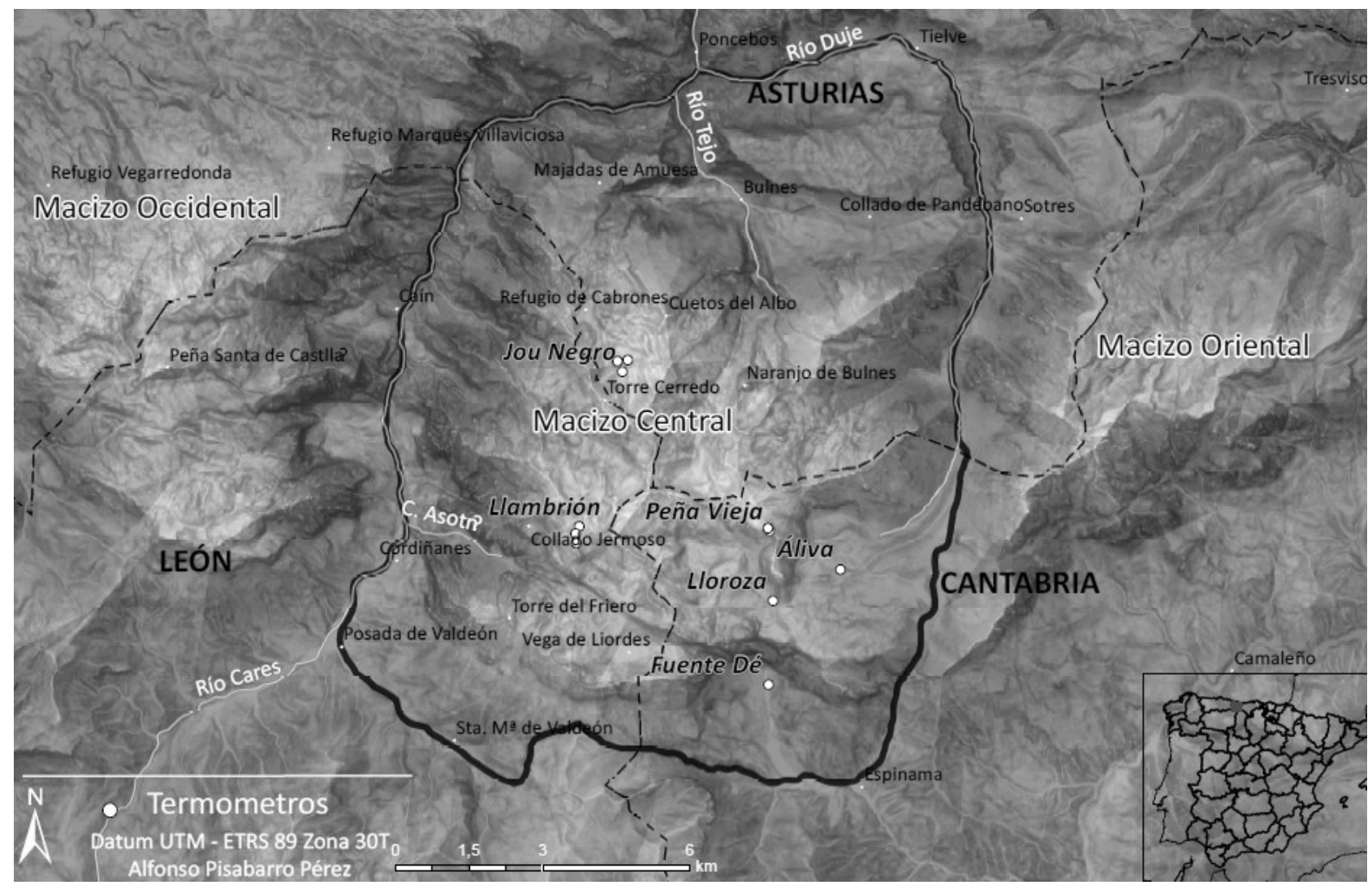

Figura 1. Emplazamiento de los termorregistradores en el Macizo Central de Picos de Europa. Figure 1. Location of microsensors in the Central Massif of Picos de Europa.

ción, como en el resto de investigaciones posteriores, se sufrieron numerosas pérdidas de datos y roturas de termorregistradores por las extremas condiciones del macizo. Bajo estas circunstancias, Serrano y González Trueba (2004) y González Trueba (2007) realizaron el análisis del régimen térmico en las faldas del grupo Peña Vieja. Pudieron distinguir tres fases en el régimen térmico anual muy marcadas. Recientemente, Pisabarro (2013) ha realizado el análisis en profundidad de los regímenes térmicos, estadísticos básicos de temperatura y las respuestas geomorfológicas que se sintetizan en el presente artículo. Si tenemos en cuenta las carencias de conocimiento que existen en el Macizo de Picos de Europa acerca del estudio térmico de los suelos, en parte consecuencia de lo abrupto y peligroso de su tránsito, gana importancia la presente investigación de base. Con ella se pretenden obtener los parámetros aproximados en los que se pudieran fundamentar futuras investigaciones relacionadas con las respuestas geomorfológicas derivadas de tres de los principales procesos térmicos. El primero de ellos es el régimen térmico anual en diferentes puntos del macizo bajo diferentes condiciones topoclimáticas; en segundo lugar estaría la magnitud y duración de la penetración de la onda de frío a través del índice de helada y la duración de temperaturas por debajo de $0^{\circ} \mathrm{C}$, y, por último, el número y distribución temporal de los ciclos de hielo y deshielo.

\section{Metodología}

Los datos de temperaturas fueron obtenidos mediante microsensores térmicos I-Bottom UTL-Geotest AG (Universal Temperature Logger) data-logger con precisión centesimal, enterrados a $10 \mathrm{~cm}$ de profundidad. Estos permiten el control del régimen térmico del suelo, mejorando las potencialidades de las mediciones BTS para suelos permanentemente helados o estacionales. Se han registrado datos de diferentes años entre 2003 y 2007 con una frecuencia de 2 horas (Tabla 1) y un nivel de error de $0,05^{\circ} \mathrm{C}$. La elección de los puntos de registro de las temperaturas responde en primer lugar a un escalonamiento altimétrico y en segundo lugar a un estudio de detalle para los lugares previsiblemente más fríos por la existencia de hielo fósil, caso de los heleros del Llambrión y del Jou Negro (Figura 2).

Desafortunadamente en el momento de la recogida, varios micro sensores contenían series más cortas de datos o desaparecieron como consecuencia de averías producidas por las condiciones meteorológicas y geodinámicas a los que estuvieron expuestos. En total se recuperaron 12 termómetros con series largas y representativas, repartidos en 6 lugares de estudio entre los 1.115 y 2.535 m.s.n.m. de altitud perteneciendo 9 de ellos al piso crionival. El tratamiento de los datos ha consistido en la obten- 


\section{4 • A. PISABARRO PÉREZ, E. SERRANO CAÑADAS Y J.J. GONZÁLEZ TRUEBA}

Tabla 1: Características de la localización de los datos tomados.

Table 1: Features of location data taken.

\begin{tabular}{|c|c|c|c|c|c|c|}
\hline \multicolumn{2}{|c|}{ Llambrión } & Año & Coordenadas & Jou Negro & Año & Coordenadas \\
\hline Altitud & 2.535 & 20/9/05-14/5/2007 & $43^{\circ} 10^{\prime} 24.97^{\prime \prime} \mathrm{N} \mid 4^{\circ} 51^{\prime} 18.65^{\prime \prime} \mathrm{W}$ & 2.155 & 20/9/2005-14/7/2007 & $43^{\circ} 12^{\prime} 7.84^{\prime \prime} \mathrm{N} \mid 4^{\circ} 51^{\prime} 7.59^{\prime \prime} \mathrm{W}$ \\
\hline Profundidad & $10 \mathrm{~cm}$ & Orientación & $\mathrm{N}$ & $10 \mathrm{~cm}$ & Orientación & $\mathrm{N}$ \\
\hline Tipo & UTL-1 & Intérvalo & 2 horas & UTL-1 & Intérvalo & 2 horas \\
\hline Sustrato & \multicolumn{3}{|c|}{ Calizas } & \multicolumn{3}{|c|}{ Calizas } \\
\hline Forma & \multicolumn{3}{|c|}{ Umbral con material morrénico } & \multicolumn{3}{|c|}{ Morrena, zona inferior } \\
\hline Depósito & \multicolumn{3}{|c|}{ Till derrubiado } & \multicolumn{3}{|c|}{ Till } \\
\hline \multicolumn{2}{|c|}{ Jou Trasllambrión } & Año & Coordenadas & Lloroza & Año & Coordenadas \\
\hline Altitud & 2.490 & 20/9/05-14/5/2007 & $43^{\circ} 10^{\prime} 33.27^{\prime \prime} \mathrm{N} \mid 4^{\circ} 51^{\prime} 14.65^{\prime \prime} \mathrm{W}$ & 1.865 & 20/9/2005-14/7/2007 & $43^{\circ} 9^{\prime} 35.4^{\prime \prime} \mathrm{N} \mid 4^{\circ} 48^{\prime} 43.32^{\prime \prime} \mathrm{W}$ \\
\hline Profundidad & $10 \mathrm{~cm}$ & Orientación & $\mathrm{N}$ & $10 \mathrm{~cm}$ & Orientación & $\mathrm{N}$ \\
\hline Tipo & UTL-1 & Intérvalo & 2 horas & UTL-1 & Intérvalo & 2 horas \\
\hline Sustrato & \multicolumn{3}{|c|}{ Calizas } & \multicolumn{3}{|c|}{ Calizas } \\
\hline Forma & \multicolumn{3}{|c|}{ Umbral con material morrénico } & \multicolumn{3}{|c|}{ Morrenas del tardiglaciar } \\
\hline Depósito & \multicolumn{3}{|c|}{ Till derrubiado } & \multicolumn{3}{|c|}{ Till } \\
\hline \multicolumn{2}{|c|}{ Jou Trasllambrión } & Año & Coordenadas & Áliva & Año & Coordenadas \\
\hline Altitud & 2.360 & 20/9/05-14/5/2007 & $43^{\circ} 10^{\prime} 44.73^{\prime \prime} \mathrm{N} \mid 4^{\circ} 51^{\prime} 11.72^{\prime \prime} \mathrm{W}$ & 1.735 & 20/9/2005-14/7/2007 & $43^{\circ} 11^{\prime} 39.71^{\prime \prime} \mathrm{N} \mid 4^{\circ} 46^{\prime} 17.06^{\prime \prime} \mathrm{W}$ \\
\hline Profundidad & $10 \mathrm{~cm}$ & Orientación & $\mathrm{N}$ & $10 \mathrm{~cm}$ & Orientación & $\mathrm{N}$ \\
\hline Tipo & UTL-1 & Intérvalo & 2 horas & UTL-1 & Intérvalo & 2 horas \\
\hline Sustrato & \multicolumn{3}{|c|}{ Calizas } & \multicolumn{3}{|c|}{ Pizarras (Fm Lebeña) } \\
\hline Forma & \multicolumn{3}{|c|}{ Talud de derrubios } & \multicolumn{3}{|c|}{ Ladera regularizada, bloques dispersos } \\
\hline Depósito & \multicolumn{3}{|c|}{ Coluvión de gruesos } & \multicolumn{3}{|c|}{ Coluvión de finos } \\
\hline \multicolumn{2}{|c|}{ Jou Negro } & Año & Coordenadas & Fuente Dé & Año & Coordenadas \\
\hline Altitud & 2.190 & $13 / 9 / 2006-25 / 12 / 07$ & $43^{\circ} 12^{\prime} 7.43^{\prime \prime} \mathrm{N} \mid 4^{\circ} 51^{\prime} 9.17^{\prime \prime} \mathrm{W}$ & $1.115 \mathrm{~m}$ & 20/9/2005-14/7/2007 & $43^{\circ} 8^{\prime} 56.76^{\prime \prime} \mathrm{N} \mid 4^{\circ} 48^{\prime} 35.277^{\prime \prime} \mathrm{W}$ \\
\hline Profundidad & superficie & Orientación & $\mathrm{N}$ & $10 \mathrm{~cm}$ & Orientación & $\mathrm{N}$ \\
\hline Tipo & UTL-1 & Intérvalo & 4 horas & UTL-1 & Intérvalo & 2 horas \\
\hline Sustrato & \multicolumn{3}{|c|}{ Calizas } & \multicolumn{3}{|c|}{ Calizas } \\
\hline Forma & \multicolumn{3}{|c|}{ Deslizamiento de la morrena con suelos ordenados } & & Cono mixto, tor & encial-aludes \\
\hline Depósito & & Till retrabajado por & rocesos periglaciares & & Aluvial, grues & s con matriz \\
\hline Jou N & & Año & Coordenadas & Peña Vieja & Año & Coordenadas \\
\hline Altitud & 2.190 & $13 / 9 / 2006-25 / 12 / 07$ & $43^{\circ} 12^{\prime} 7.43^{\prime \prime} \mathrm{N} \mid 4^{\circ} 51^{\prime} 9.17^{\prime \prime} \mathrm{W}$ & 2.510 & $21 / 09 / 03-29 / 10 / 04$ & $43^{\circ} 10^{\prime} 29.23^{\prime \prime} \mathrm{N} \mid 4^{\circ} 48^{\prime} 38.09^{\prime \prime} \mathrm{W}$ \\
\hline Profundidad & $10 \mathrm{~cm}$ & Orientación & $\mathrm{N}$ & $10 \mathrm{~cm}$ & Orientación & NW \\
\hline Tipo & UTL-1 & Intérvalo & 4 horas & UTL-1 & Intérvalo & 2 horas \\
\hline Sustrato & & & izas & & Cali & \\
\hline Forma & & eslizamiento de la mor & ena con suelos ordenados & & Canal donde se inicic & cono de derrubios \\
\hline Depósito & & Till retrabajado por & rocesos periglaciares & Coluvión & n, derrubios de gravedad & nnos y homométrico con matriz \\
\hline Jou $N_{c}$ & & Año & Coordenadas & Peña Vieja & Año & Coordenadas \\
\hline Altitud & 2.205 & 20/9/2005-14/7/2007 & $43^{\circ} 12^{\prime} 10.05^{\prime \prime} \mathrm{N} \mid 4^{\circ} 50^{\prime} 59.68^{\prime \prime} \mathrm{W}$ & 2.325 & $21 / 09 / 03-10 / 05 / 2005$ & $43^{\circ} 10^{\prime} 31.21^{\prime \prime} \mathrm{N} \mid 4^{\circ} 48^{\prime} 43.82^{\prime \prime} \mathrm{W}$ \\
\hline Profundidad & $10 \mathrm{~cm}$ & Orientación & $\mathrm{N}$ & $10 \mathrm{~cm}$ & Orientación & NW \\
\hline Tipo & UTL-1 & Intérvalo & 2 horas & UTL-1 & Intérvalo & 2 horas \\
\hline Sustrato & & & izas & & Cali & \\
\hline Forma & & lud de derrubios que er & laza con la morrena lateral & & Depresión kárstica con $\mathrm{r}$ & cubrimiento de ladera \\
\hline Depósito & Derrul & ios de gravedad muy h & terométrico, estructura abierta & Coluv & ión, derrubios de graved & d heterométricos con matriz \\
\hline
\end{tabular}



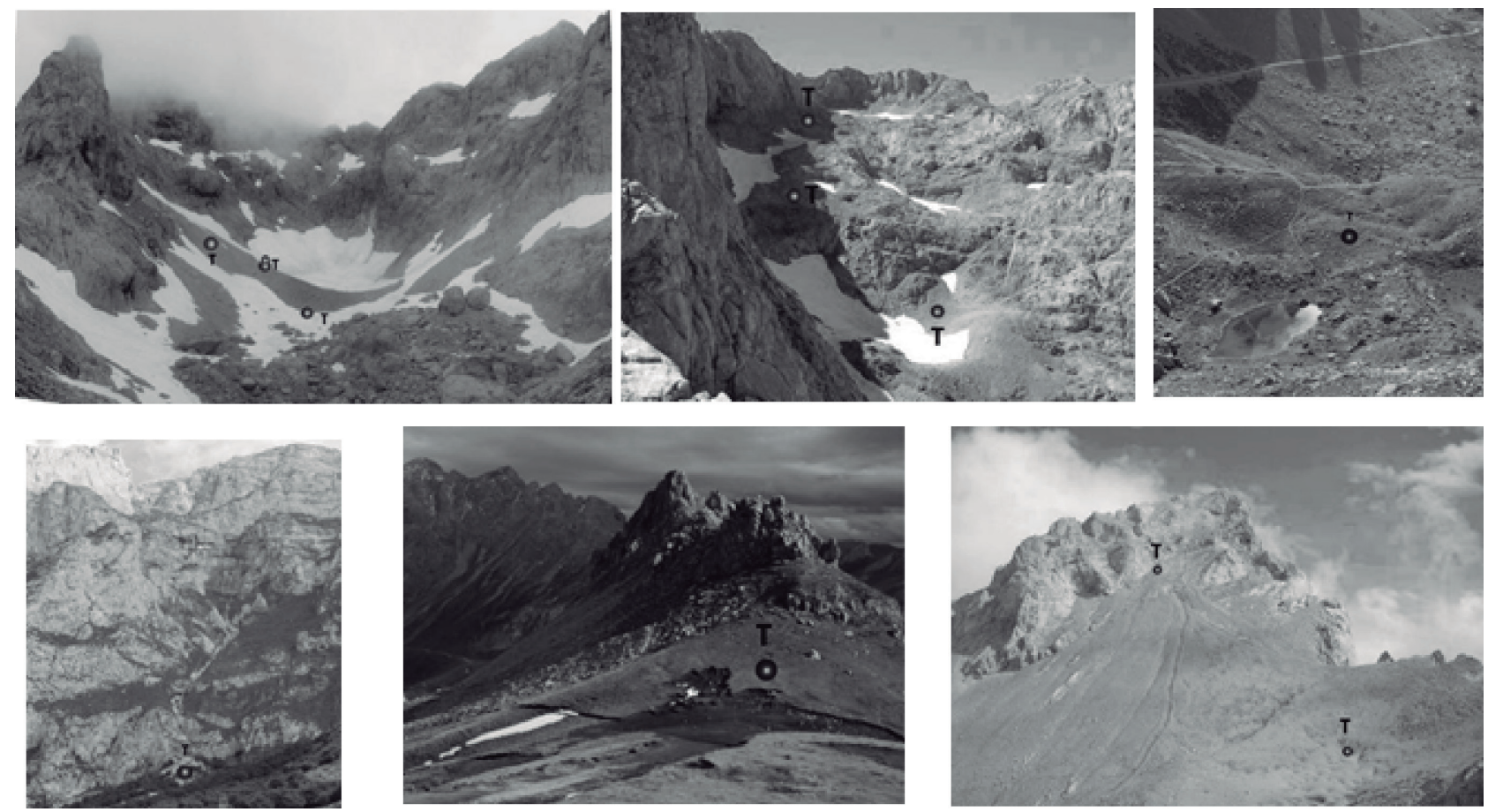

Figura 2. Emplazamiento de los microsensores de izquierda a derecha y de arriba a abajo: Jou Negro, Jou Trasllambrión, Lloroza, Fuente Dé, Áliva y Peña Vieja.

Figure 2. Location of microsensors from left to right and top to bottom: Jou Negro, Jou Trasllambrión, Lloroza, Fuente Dé, Aliva and Peña Vieja.

ción de estadísticos y de los parámetros más representativos como son el índice de helada ${ }^{1}$ y el número de días de hielo-deshielo a partir de los datos de temperaturas ${ }^{2}$. Se ha tomado como referencia metodológica el estudio del régimen térmico realizado por Delaloye (2004).

\section{Resultados}

\subsection{Fases y ciclos térmicos}

En el análisis de los gráficos de temperatura media diaria (Figura 3) se pueden distinguir hasta cuatro fases térmicas de duración variable en función de las peculiaridades topoclimáticas del emplazamiento. Estas fases se aprecian mejor a partir de 1.700 m.s.n.m. por la existencia de un manto nival grueso y prolongado. El criterio para distinguirlas viene determinado por el comportamiento de las gráficas de temperaturas medias diarias que se describen a continuación. El análisis de cada una de ellas muestra cuatro fases térmicas diferenciadas:
Fase de altas temperaturas (fase 1). Prosigue a la fusión de la nieve entre abril y julio. Esta fase se extiende hasta principios de otoño cuando el suelo está en contacto directo con la atmósfera y la temperatura nunca es inferior de los $0^{\circ} \mathrm{C}$.

Fase de transición verano-invierno (fase 2). Es un intervalo en el cual las temperaturas se acercan o incluso descienden por debajo de los $0^{\circ} \mathrm{C}$. Se aprecia una gran inestabilidad térmica propiciada por las primeras nevadas y descensos importantes de las temperaturas atmosféricas. La inestabilidad se debe a que el manto nival es aún efímero en estas fechas quedando el suelo expuesto a la influencia atmosférica y, por lo tanto, a un mayor número de ciclos de hielo y deshielo (Figura 4). Esta es la fase menos definida de todas. El cambio de comportamiento se produce a finales de septiembre o principios de octubre por encima de los 2.000 m.s.n.m. Por debajo de esa cota el comienzo varía según el grosor del manto nival y de lo efímero que pueda ser. Por esta razón Áliva, a menor altitud que Lloroza, tiene esta fase menos definida. Las paredes, crestas, y umbrales donde no se acumula la nieve

${ }^{1}$ Índice de helada (Ih): $I h=\int_{t 0}^{t 1} T|d T| ; T i<0^{\circ} C=\sum_{i=1}^{n}|T i| ; T i<0^{\circ} C$ (Fengquing y Yanwei, 2011) El índice de helada ha sido necesario para comparar profundidades de hielo estacional (French, 2007) así como su magnitud.

${ }^{2}$ En Pisabarro (2013) se incluye el análisis previo de las temperaturas medias, mínimas, máximas, amplitudes térmicas y oscilaciones diarias que han servido de base para el presente artículo. 

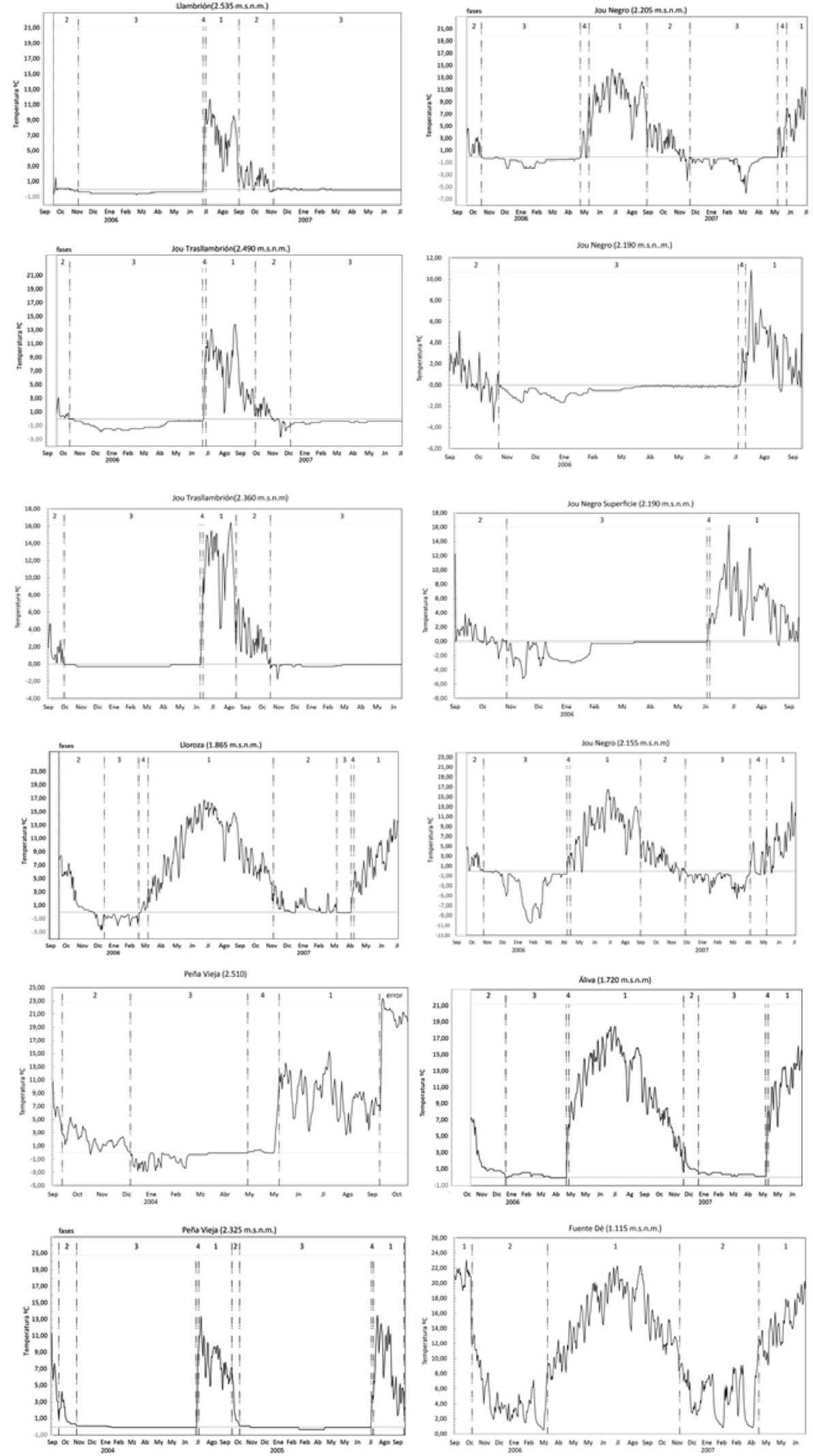

Figura 3. Fases y regímenes térmicos del suelo en las distintas localizaciones estudiadas. Figure 3. Phases and ground thermal regimes in different researched locations 


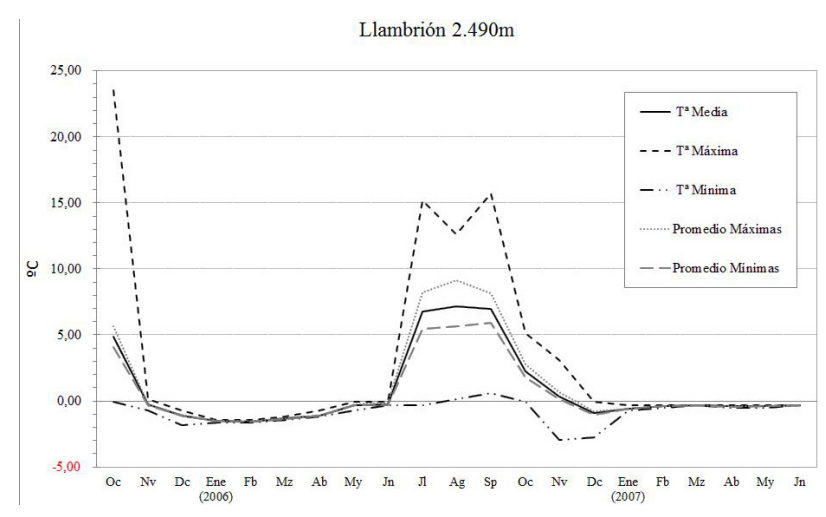

Figura 4. Régimen térmico anual con datos mensuales del termorregistrador situado en el Jou Trasllambrión a 2.490 m.s.n.m.

Figure 4. Annual thermal regime with monthly data microsensor located in the Jou Trasllambrión to 2,490 m.a.s.l.

permanecen con este comportamiento hasta la llegada de la primavera.

Fase de equilibrio isotérmico (fase 3 ). Cuando la acumulación de nieve es suficiente para aislar el suelo de la atmósfera, comienza un largo período invernal y pri- maveral de equilibrio isotérmico. Las temperaturas acusan una estabilidad cuasi absoluta y la protección del manto nival hace que máximas y mínimas coincidan durante días, semanas o incluso meses.

El inicio de la fase 3 viene determinado por la proximidad de las temperaturas máximas y mínimas diarias, siempre por debajo de $\operatorname{los} 0^{\circ} \mathrm{C}$. La duración varía en función de las primeras nevadas, del espesor de nieve capaz de almacenarse y de la rapidez de la fusión nival en base a los condicionantes topoclimáticos del lugar. Los termorregistradores marcan un intervalo de duración de 3 a 9 meses donde bajo el manto nival la estabilidad térmica es absoluta. Esto puede enmascarar microprocesos de hielo y deshielo como el «zero courtain effect». Investigaciones en el Ártico indican que el fenómeno podría ser universal (Outcalt et al., 1990). Esta fase es la que permite afirmar la existencia generalizada de suelos helados estacionales en algunos puntos del Macizo Central de Picos de Europa (Tabla 2), en general desde los 1.700-2.000 m.s.n.m. Los suelos helados estacionales según la definición de French (2007), solo hacen referencia a los suelos en los que se congela el agua procedente del manto nival $\left(0^{\circ} \mathrm{C}\right)$ durante un período prolongado del invierno. La duración del mismo está sujeta a discusión científica. En lo que respecta a las diferencias de temperaturas entre años se pueden observar contradicciones en cuanto a la fase de

Tabla 2: Duración y magnitud de la fase de equilibrio térmico.

Table 2: Duration and magnitude of the thermal equilibrium phase.

\begin{tabular}{|c|c|c|c|c|c|c|c|}
\hline Termómetro & Altitud & Inicio & Fin & Temperatura ${ }^{\circ} \mathrm{C}$ & $\begin{array}{c}\text { Suelos helados } \\
\text { estacionales }\end{array}$ & Localización & Formación superficial \\
\hline Llambrión & 2.535 & Noviembre/Diciembre & Julio & $\mathrm{De}-0,5$ a 0 & Posible & $\begin{array}{c}\text { Umbral con till } \\
\text { derrubiado }\end{array}$ & Till derrubiado \\
\hline Peña Vieja & 2.510 & Marzo & Mayo & De -1 a 0 & Posible & $\begin{array}{l}\text { Cono de derrubios con } \\
\text { finos y matriz }\end{array}$ & $\begin{array}{c}\text { Coluvión, finos con } \\
\text { matriz }\end{array}$ \\
\hline $\begin{array}{c}\text { Jou } \\
\text { Trasllambrión }\end{array}$ & 2.490 & Enero & Julio & $\mathrm{De}-1,5 \mathrm{a}-0,3$ & Sí & $\begin{array}{l}\text { Umbral con till } \\
\text { derrubiado }\end{array}$ & Till derrubiado \\
\hline $\begin{array}{c}\text { Jou } \\
\text { Trasllambrión }\end{array}$ & 2.360 & Noviembre/Diciembre & Julio & De $-0,5$ a 0 & Posible & $\begin{array}{l}\text { Talud de derrubios } \\
\text { gruesos }\end{array}$ & Coluvión de gruesos \\
\hline Peña Vieja & 2.325 & Noviembre & Julio & De $-0,2$ a 0,2 & Posible & $\begin{array}{l}\text { Depresión kárstica con } \\
\text { recubrimiento de ladera }\end{array}$ & $\begin{array}{c}\text { Coluvión, derrubios } \\
\text { heterométricos con matriz }\end{array}$ \\
\hline Jou Negro & 2.205 & Noviembre/Diciembre & Mayo & De $-1,5$ a $-0,5$ & Sí & Talud de derrubios & Derrubios de gravedad \\
\hline $\begin{array}{l}\text { Jou Negro } \\
\text { (Superficie) }\end{array}$ & 2.190 & Diciembre & Junio & $\mathrm{De}-2,5 \mathrm{a}-0,1$ & Sí & $\begin{array}{l}\text { Deslizamiento de morrena } \\
\text { con suelos ordenados }\end{array}$ & $\begin{array}{c}\text { Till retrabajado por } \\
\text { procesos periglaciares }\end{array}$ \\
\hline Jou Negro & 2.190 & Diciembre & Julio & $\mathrm{De}-1,1 \mathrm{a}-0,1$ & Sí & $\begin{array}{l}\text { Deslizamiento de morrena } \\
\text { con suelos ordenados }\end{array}$ & $\begin{array}{c}\text { Till retrabajado por } \\
\text { procesos periglaciares }\end{array}$ \\
\hline Jou Negro & 2.155 & Noviembre/Diciembre & Abril & $\mathrm{De}-8,5$ a 0 & Sí & $\begin{array}{c}\text { Morrena lateral, zona } \\
\text { inferior }\end{array}$ & Till \\
\hline Lloroza & 1.865 & Diciembre/ Enero & $\begin{array}{c}\text { Marzo/ } \\
\text { Abril }\end{array}$ & De $-0,7$ y 0,5 & Posible & Morrenas del tardiglaciar & Till \\
\hline Áliva & 1.720 & Enero & $\begin{array}{l}\text { Abril/ } \\
\text { Mayo }\end{array}$ & De 0 a 0,5 & Posible & $\begin{array}{l}\text { Coluvión con finos, } \\
\text { bloques dispersos }\end{array}$ & Coluvión de finos \\
\hline Fuente Dé & 1.115 & & & & Imposible & $\begin{array}{l}\text { Cono de gruesos con } \\
\text { matriz, torrencial-aludes }\end{array}$ & $\begin{array}{l}\text { Aluvial, gruesos con } \\
\text { matriz }\end{array}$ \\
\hline
\end{tabular}


estabilidad invernal. Por lo general, en el año 2006 la onda de frio tuvo mayor penetración, sin embargo en los microsensores del Jou Trasllambrión (2.490 m.s.n.m.) y en el Jou Negro (2.205 m.s.n.m.), en el invierno del año 2007 fue cuando se alcanzaron las temperaturas mínimas. Estas contradicciones pueden responder a los siguientes factores; una intensidad mayor de las temperaturas mínimas en ese mismo año, la inestabilidad de la nieve y la influencia atmosférica, o bien al ascenso por convección de flujos de aire desde los heleros fósiles contiguos. En Fuente Dé se han descartado los suelos helados en base a las condiciones topoclimáticas del lugar en un abrigo con elevadas temperaturas atmosféricas.

Fase de fusión (fase 4). Es un episodio muy rápido debido a la rápida fusión de la nieve y el registro inmediato del régimen atmosférico. Se produce cuando el manto nival ha adelgazado y es lo suficientemente fino como para transmitir las variaciones térmicas de la atmósfera. La temperatura del suelo asciende de $\operatorname{los} 0^{\circ} \mathrm{C}$ a temperaturas veraniegas en un solo día en 10 de las 12 localizaciones estudiadas. Este episodio se produce entre mayo y julio dependiendo de las condiciones topoclimáticas. La desaparición tan tardía del manto nival impide que se produzcan nuevos fenómenos de congelación y ciclos de hielo/deshielo, ascendiendo los registros rápidamente al entorno de los $10-20^{\circ} \mathrm{C}$.

\subsection{Magnitud del enfriamiento}

El número de días anuales con temperatura del suelo por debajo de $0^{\circ} \mathrm{C}$ supera el $50 \%$ para el $75 \%$ de las localizaciones estudiadas. No obstante, durante el período de máxima estabilidad térmica a finales del invierno, el umbral $-2{ }^{\circ} \mathrm{C}$ se considera el valor mínimo del intervalo tér- mico invernal (Haeberli, 1973; French, 2007) para considerar la existencia de permafrost esporádico.

Hay tres emplazamientos en los que se superan los $-2{ }^{\circ} \mathrm{C}$ de forma significativa, sobretodo en el Jou Negro. No obstante, estos datos son insuficientes para establecer la existencia de permafrost, ya que estas temperaturas se mantienen durante un breve período de tiempo (Tabla 3). La existencia del helero fósil junto a la morrena donde se han situado los termorregistradores con los índices de helada más elevados y de dos «frost mounts» parece indicar que puedan estar afectados por elementos helados en profundidad o por movimientos convectivos de aire (González Trueba, 2007).

El análisis de la relación entre el índice anual de helada y la altitud (Figura 5) indica la independencia de las dos variables ya que presenta una bondad de ajuste $\mathrm{R}^{2}$ muy baja por lo que se deduce que influyen mucho más otros factores topoclimáticos, en especial la cubierta nival.

Los registros del Jou Negro son los que tienen un mayor índice de helada con mucha diferencia a pesar de no ser los obtenidos a mayor altitud. Por debajo de los 2.000 m.s.n.m., el índice de helada es muy bajo en Áliva y Lloroza y nulo en Fuente Dé. En el Llambrión tampoco se registra el mayor índice de helada en el termorregistrador de mayor altitud. Sí en el caso de Peña Vieja. Las largas series de hasta $65 \%$ de días por debajo de $0^{\circ} \mathrm{C}$ del Llambrión y de Peña Vieja y su bajo índice de helada se deben a que la estabilidad invernal se prolonga buena parte del año con registros muy cercanos a los $0^{\circ} \mathrm{C}$ (en el entorno de los $-0,3^{\circ} \mathrm{C}$ ) como consecuencia de una gruesa capa de nieve, estable hasta el comienzo del verano.

Se puede dar un orden de magnitud en cuanto a la profundidad que alcanza la helada mediante la fórmula planteada por Washburn (1979). Tiene en cuenta el calor la-

Tabla 3: Tabla resumen del número de días de congelación e índice de helada.

Table 3: Summary table of the number of days of freezing and freeze index.

\begin{tabular}{|c|c|c|c|c|c|c|c|}
\hline & Altitud & Año completo & ${\text { Días } \mathbf{T}^{\mathbf{a}}<\mathbf{0}^{\mathbf{0}} \mathbf{C}}$ & $\mathbf{\%}$ & Días $^{\mathbf{a}}<-\mathbf{2}^{\mathbf{0}} \mathbf{C}$ & $\mathbf{\%}$ & Í. de helada (Ih) $^{\mathbf{2}}$ \\
\hline Llambrión & 2.535 & 2006 & 217 & 59,5 & 0 & 0,0 & 84,77 \\
\hline Peña Vieja & 2.510 & $10 / 2003-10 / 2004$ & 146 & 40,0 & 17 & 4,7 & 95,62 \\
\hline Jou Trasllambrión & 2.490 & 2006 & 234 & 64,1 & 2 & 0,5 & 218,71 \\
\hline Jou Trasllambrión & 2.360 & 2006 & 238 & 65,2 & 0 & 0,0 & 58,83 \\
\hline Peña Vieja & 2.325 & 2004 & 230 & 63,0 & 0 & 0,0 & 19,74 \\
\hline Jou Negro & 2.205 & 2006 & 121 & 33,2 & 2 & 0,5 & 112,75 \\
\hline Jou Negro (Superficie) & 2.190 & $11 / 2005-11 / 2006$ & 277 & 75,9 & 1 & 0,3 & 138 \\
\hline Jou Negro & 2.190 & $11 / 2005-11 / 2006$ & 238 & 65,2 & 65 & 17,8 & 235 \\
\hline Jou Negro & 2.155 & 2006 & 176 & 48,2 & 63 & 17,3 & 461,34 \\
\hline Lloroza & 1.865 & 2006 & 79 & 21,6 & 2 & 0,5 & 55,17 \\
\hline Áliva & 1.720 & 2005 & 34 & 9,3 & 0 & 0,0 & 2,64 \\
\hline Fuente Dé & 1.115 & 2006 & 0 & 0,0 & 0 & 0,0 & 0 \\
\hline
\end{tabular}




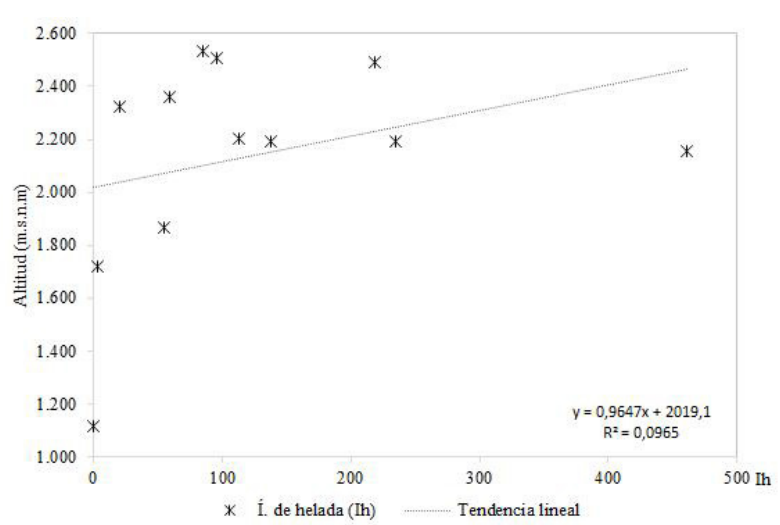

Figura 5. Índice de helada en función de la altitud. Figure 5. Freeze index depending on the altitude.

tente del agua a volumen constante $\left(\mathrm{C}_{\mathrm{L}}\right)$, la conductividad térmica del suelo $(\mathrm{K})$ y el índice de helada $\left(\mathrm{I}_{\mathrm{h}}\right)^{3}$.

Se estima que la conductividad térmica de una caliza normal es de $1,4 \mathrm{~W}^{*} \mathrm{~m}^{-1 *} \mathrm{~K}^{-1}$ mientras que la de la arcilla que aparece en la matriz es de $1,5 \mathrm{~W}^{*} \mathrm{~m}^{-1} * \mathrm{~K}^{-1}$. El calor latente a $0^{\circ} \mathrm{C}$ como promedio obtenido en condiciones ideales es de $79 \mathrm{Kcal}$. Aplicando estos datos a los dos emplazamientos con mayor índice de helada donde la formación superficial es till se aplica una conductividad media de caliza y arcilla $\left(1,45 \mathrm{~W}^{*} \mathrm{~m}^{-1 *} \mathrm{~K}^{-1}\right)$.

- Jou Negro (2.155 m.s.n.m.) (Formación de till e índice de helada de 461,34).

Profundidad de la helada: 0,40 metros. Sumando los $\sim 10 \mathrm{~cm}$ a los que está enterrado el termómetro serían $\sim \mathbf{0 , 5 0}$ metros en condiciones ideales.

- Llambrión (2.490 m.s.n.m.) (Formación de till e índice de helada de 218,71).

Profundidad de la helada $=0,28$ metros. Sumando los $10 \mathrm{~cm}$ a los que está enterrado el termómetro serían $\sim \mathbf{0 , 3 8}$ metros en condiciones ideales.

En ambos casos, el tipo de suelo es de composición heterométrica, lo que permite la penetración de movimientos convectivos desde la parte inferior de las morrenas, donde se sitúan heleros fósiles, lo cual implicaría cambios en la profundidad de la helada. En el caso del Jou Negro existen dos lóbulos de gelifluxión y hasta tres frost mounds visibles, lo que corrobora el elevado índice de helada y la penetración de la misma en el suelo.

\subsection{Ciclos de hielo/deshielo}

Los ciclos de hielo-deshielo son muy variables y dependen de la existencia de una cubierta importante de nie- ve. Los ciclos son más numerosos en fuertes pendientes, crestas, lugares ventosos o extraplomos, donde se producen los procesos periglaciares más intensos por la inexistencia de la protección del manto nival. Por el contrario los ciclos de hielo/deshielo son menos intensos en los lugares que permiten fácilmente la acumulación de la nieve y, por lo general, son inferiores a 10 días al año. Generalmente se extiende más el período de hielo-deshielo en otoño. Este suele durar unos 5-6 días hasta que se cubren los suelos con las primeras nevadas importantes. En primavera-verano lo normal es que solo exista un día de hielo-deshielo por encima de los 1.800 - 2.000 m.s.n.m. que ocurre finalizando la primavera o incluso avanzado el verano dependiendo del grosor de manto nival acumulado. En este caso, la fusión de la nieve coincide con temperaturas atmosféricas muy altas, lo que produce un incremento repentino de temperaturas en el suelo. Generalmente, los sitios con un mayor número de ciclos y más tempranos coinciden con lugares de solana. También puede ocurrir con precipitaciones de carácter tormentoso muy frecuentes en el macizo. En los registros de los períodos anuales elegidos se puede apreciar disparidad de situaciones. El Jou Negro es un lugar propicio para los fenómenos de hielo/deshielo donde los registros varían desde los 10 a los 40 ciclos. En el Llambrión, Peña Vieja, Lloroza y Áliva, lugares con una mayor estabilidad, acumulan entre 6 y 15 días.

La distribución mensual de los ciclos de hielo/deshielo (Tabla 4) muestra que la mayoría de estos ciclos ocurren a final de otoño (fase 2 del régimen térmico). El grosor del manto nival en esas fechas es escaso aún y se alternan situaciones atmosféricas cálidas y frías. Especialmente aclaratorios son los ciclos de julio, momento en el que la fusión de la nieve significa una subida repentina de $20^{\circ} \mathrm{C}$ en las temperaturas en un solo día. Los ciclos percibidos en agosto, septiembre y octubre responden al enfriamiento de las mínimas nocturnas que en estas fechas ya pueden ser inferiores a $\operatorname{los} 0^{\circ} \mathrm{C}$.

\section{Discusión}

Los estudios sobre régimen térmico en la alta montaña de la Cordillera Cantábrica, y en general en la Península Ibérica, muestran resultados difíciles de comparar debido a que cada emplazamiento posee características únicas. Sin ir más lejos, la nieve regula los ciclos térmicos, pero su desigual distribución y acumulación imposibilita establecer umbrales cuantitativos sólidos para determinar las fases en cada lugar del macizo. Sin embargo, sí que se pueden advertir cambios de tendencia. Por ello, las cuatro fases térmicas definidas en este artículo son sin lugar a dudas fruto de debate. González Trueba (2007) define hasta cinco fases en su análisis del Grupo Peña

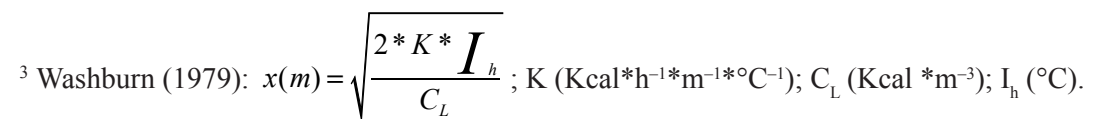


Tabla 4: Distribución mensual de los ciclos de hielo/deshielo.

Table 4: Monthly distribution of freeze / thaw.

\begin{tabular}{|c|c|c|c|c|c|c|c|c|c|c|c|c|c|c|c|}
\hline & & Ene & Feb & Marzo & Abril & Mayo & Junio & Julio & Ago & Sept & Oct & Nov & Dic & Total & Año completo útil \\
\hline Llambrión & 2.535 & & & & & & & 1 & & 1 & 2 & 2 & 9 & $\mathbf{1 5}$ & 2006 \\
\hline Peña Vieja & 2.510 & & & & & 5 & & & & & & 3 & 1 & $\mathbf{9}$ & $10 / 2003-10 / 2004$ \\
\hline Jou Trasllambrión & 2.490 & & & & & & & 1 & & & 1 & 6 & & $\mathbf{8}$ & 2006 \\
\hline Jou Trasllambrión & 2.360 & & & & & & & 1 & 2 & & & & & $\mathbf{3}$ & 2006 \\
\hline Peña Vieja & 2.325 & 13 & 1 & & & & & 1 & & & & & & $\mathbf{1 5}$ & 2004 \\
\hline Jou Negro & 2.205 & & & & & 5 & & & & & & 3 & 2 & $\mathbf{1 0}$ & 2006 \\
\hline Jou Negro (Superficie) & 2.190 & & & & & & & 1 & 2 & 5 & 8 & 14 & 4 & $\mathbf{3 4}$ & $11 / 2005-11 / 2006$ \\
\hline Jou Negro & 2.190 & 4 & & & & & & & 4 & 4 & 10 & 14 & 4 & $\mathbf{4 0}$ & $11 / 2005-11 / 2006$ \\
\hline Jou Negro & 2.155 & & & & 4 & 4 & & & & & & 6 & 4 & $\mathbf{1 8}$ & 2006 \\
\hline Lloroza & 1.865 & & & 7 & & & & & & & & & 1 & $\mathbf{8}$ & 2006 \\
\hline Áliva & 1.720 & 1 & 1 & 3 & 1 & & & & & & & & & $\mathbf{6}$ & 2005 \\
\hline Fuente Dé & 1.115 & & & & & & & & & & & & & $\mathbf{0}$ & 2006 \\
\hline \multicolumn{2}{c}{ Total } & & $\mathbf{1 8}$ & $\mathbf{2}$ & $\mathbf{1 0}$ & $\mathbf{5}$ & $\mathbf{1 4}$ & $\mathbf{0}$ & $\mathbf{5}$ & $\mathbf{8}$ & $\mathbf{1 0}$ & $\mathbf{2 1}$ & $\mathbf{4 8}$ & $\mathbf{2 5}$ & \\
\hline
\end{tabular}

Vieja. Separa la fase número 2 en una fase de conexión verano-otoño y otra de otoño-invierno. En su caso, se podía apreciar una fase de temperaturas en descenso sin nieve y otra con descensos más marcados de las temperaturas y efectos de la nieve. Sería incluso fácilmente asumible establecer una sexta fase a comienzos de la fase 3 en la cual existe cierta amplitud térmica previa al efecto cortina por debajo siempre de $0^{\circ} \mathrm{C}$ para los registros más fríos del Jou Negro y el Jou Trasllambrión.

La correlación de altitud y temperaturas mínimas nos sugirió que la altitud no era el factor con más incidencia en Picos de Europa, sin embargo Pellitero (2013) afirma en el macizo de Fuentes Carrionas lo contrario, aunque otorga casi el mismo peso a las orientaciones Oeste y Norte. En cuanto a la existencia de permafrost afirma que no se cumplen las condiciones para su existencia aunque advierte de la importante acción de la helada. Una acción de la helada que se plasma en el mapa mundial del permafrost y los suelos helados del National Snow and Ice Data Center que corrobora la existencia de suelos helados estacionales de forma aislada en Picos de Europa por debajo del 10\% de su superficie (Brown et al., 2014). Dos de los enclaves más propicios para la formación de suelos helados han quedado ilustrados en el presente trabajo, ya que los registros encontrados en el Jou Negro y en menor medida en el Jou Trasllambrión sí permitirían hablar de la existencia de suelos congelados.

Será importante en futuras investigaciones diferenciar si la existencia de suelos helados en invierno es el resultado de las temperaturas atmosféricas o bien se relaciona con la influencia que ejercen cuerpos helados en profundidad (Mosciki, 2008). También será necesario establecer el papel de las posibles corrientes convectivas que desplazan aire frío a la base de cada formación superficial en pendiente. Este puede ser uno de los motivos por los cuales en varios de los termómetros instalados en un mismo sector la temperatura del suelo es inversamente proporcional a la altitud. Santos González (2011) recoge que este término recibe el nombre de «chimney effect» (Von Wakonigg, 1996) aplicándolo en su caso al estudio térmico de los glaciares rocosos.

En cuanto al escaso número de ciclos de hielo y deshielo existe bastante consenso en que la nieve reduce e incluso anula estos procesos. En el macizo, los ciclos obtenidos en los años útiles solo se pueden comparar con los datos recogidos entre Noviembre de 1995 y Mayo de 1996 por Castañón y Frochoso (1998) en el Jou de Cabrones. Estos obtuvieron 52 ciclos concentrados en otoño y primavera, una cifra muy superior debido a que el termorregistrador se instaló sobre una pared rocosa. La disminución de ciclos en presencia de manto nival concuerda con el trabajo de Santos González et al. (2009) y Santos González (2011) en el que concluye que en los termorregistradores de solana se obtiene un número muy superior de ciclos debido a la ausencia de un manto nival prolongado. Por su lado, Andrés y Palacios (2010) coinciden en señalar que en los neveros más persistentes se producen menos ciclos de hielo y deshielo. También concuerda con Salvador Franch et al. (2012), ya que obtienen numerosos ciclos en un collado venteado en el cual la inestabilidad de la nieve era alta como demuestra la escasa estabilidad de la fase de equilibrio invernal que registran. En todo caso, queda por resolver la posible existencia de microprocesos de hielo y deshielo diario que dieran respuesta científica a la intensa fracturación apreciable en sectores protegidos por grandes espesores de nieve y su relación con la karstificación.

\section{Conclusiones}

El primer objetivo del trabajo era el estudio del régimen térmico bajo diferentes condiciones topoclimáticas. 
Se ha inducido que el régimen de temperaturas de los suelos en Picos de Europa viene determinado por el espesor y duración del manto nival ya que la acumulación de la nieve invernal impide la penetración de la onda de frío y calor en el suelo, anulando la amplitud térmica de 4 a 8 meses en función de las características topoclimáticas del lugar. Estas características se compondrían de orientación, radiación, pendientes, movimientos convectivos, viento, formaciones superficiales y la propia acumulación nival.

El segundo objetivo era conocer de forma aproximada la magnitud y duración de la penetración de la onda de frío a través del manto nival mediante el índice de helada, la duración de los períodos con temperaturas por debajo de $0^{\circ} \mathrm{C} \mathrm{y}-2^{\circ} \mathrm{C}$ y fórmula de Washburn. En base a ello no se pudo determinar un gradiente altitudinal de temperaturas mínimas nítido por encima de 1.700-1.800 m.s.n.m., donde los factores topoclimáticos son los que explican la aparición de suelos helados estacionales. Sí que se pudo observar que las temperaturas en determinados lugares son de varios grados bajo cero durante los meses centrales del invierno con un elevado índice de helada. En los dos puntos más fríos del macizo, junto a heleros fósiles, se alcanzaron medias invernales por debajo de $-2^{\circ} \mathrm{C}$ (Jou Negro) y de $-1{ }^{\circ} \mathrm{C}$ (Jou Trasllambrión) originando la formación de suelos helados estacionales. Sin embargo, las temperaturas medias anuales son mayores de $-2^{\circ} \mathrm{C}$ en todos los lugares estudiados, incluso de $0^{\circ} \mathrm{C}, \mathrm{y}$, por lo tanto, es poco probable la existencia de permafrost esporádico en el Macizo Central.

El tercer objetivo consistía en analizar el número y la distribución temporal de los ciclos de hielo y deshielo. Se ha determinado que noviembre es el mes con mayor número de los mismos, seguido por diciembre, coincidiendo con la penetración de las primeras masas de aire frío ártico desde el Nordeste cuando la nieve no ha llegado a cubrir completamente el suelo. Es cuando se dan las mayores amplitudes térmicas y, por lo tanto, cuando los procesos periglaciares son más intensos.

En conclusión, el estudio de las condiciones térmicas del macizo en localizaciones escalonadas puede servir de ayuda al estudio de un medio de alta montaña rocosa, deglaciada, en el que el frío y la nieve son factores claves para entender su morfodinámica. El presente estudio de base, debe servir para ayudar a futuras investigaciones a profundizar en el estudio térmico del suelo en otros puntos del macizo, con series más largas y una densa red de microsensores térmicos con el objetivo de elaborar un mapa térmico preciso de los suelos del macizo. Entre las posibles aplicaciones del mapa estaría la prevención del riesgo por desprendimiento en rutas transitadas durante los meses con mayor número de ciclos de hielo y deshielo, además de la investigación en el campo del cambio climático.

\section{Agradecimientos}

Este trabajo se ha realizado con ayuda de los proyectos CGL2010-65295/BTE (Ministerio de Economía y
Competitividad y FEDER), y OAPN 053/2010 (Ministerio de Agricultura, Alimentación y Medio Ambiente) y con el programa de Formación de Profesorado Universitario FPU13/05837 del Ministerio de Educación Cultura y Deporte.

\section{Referencias}

Andrés, N. \& Palacios, D., 2010. Cobertura nival y distribución de la temperatura en el suelo en las cumbres de la Sierra de Guadarrama. Cuadernos de Investigación Geográfica, 36 (2): 7- 36

Brown, J., Ferrians, O., Heginbottom, J.A. \& Melnikov, E., 2014. Circum-Arctic Map of Permafrost and Ground-Ice Conditions. [On Line]. Boulder, Colorado USA: National Snow and Ice Data Center. Disponible en: $<$ http://nsidc.org/ data/ggd318> [Accedido 03 de Marzo de 2015]

Castañón, J.C. \& Frochoso, M., 1998. La alta montaña cantábrica: condiciones térmicas y morfodinámicas en los Picos de Europa. En: Gómez Ortiz, A., Salvador Franch, F., Schulte, L., García Navarro A. (Eds.), Procesos biofisicos actuales en medios fríos. Publicaciones Universidad de Barcelona, 113-132, Barcelona.

Delaloye, R., 2004. Contribution à l'étude du pergélisol de montagne en zone marginale. $\mathrm{PhD}$ thesis, Fac. Sciences, Univ. Fribourg, GeoFocus, 10.

Fengqing, J. \& Yanwei, Z., 2011. Freezing and thawing index. En Singh, V.P., Singh, P., Haritashya, U.K. (Eds.). Encyclopedia of snow, ice and glaciers, Springer, 301 pp., Dordrecht - Netherlands. http://dx.doi.org/10.1007/978-90-481-2642-2 16

French, H.M., 2007. The periglacial environment. Wiley, 478 pp., Chichester -United Kingdom. http://dx.doi. org/10.1002/9781118684931

González González, M., 2014. La alta montaña periglaciar en el Pirineo Central español: procesos, formas y condiciones ambientales. Tesis Doctoral. Departamento de Geografía. Universidad de Málaga, Málaga.

González Trueba, J.J., 2007. El macizo central de los Picos de Europa: Geomorfología y sus implicaciones geoecológicas en la alta montaña cantábrica. Tesis Doctoral. Departamento de Geografía, Urbanismo y Ordenación del Territorio, Universidad de Cantabria, Santander.

González Trueba, J.J. \& Serrano, E., 2008. Picos de Europa: El macizo de los Urrielles. En: Ruiz Flaño, P., Serrano, E., Poblete, M.A., Ruiz Fernández, J. (Eds.) De Castilla Al Mar. La naturaleza del paisaje en la Montaña Cantábrica. Asociación de Geógrafos Españoles (AGE), Universidad de Valladolid, Universidad de Oviedo, 69-98, Valladolid.

González Trueba, J.J. \& Serrano, E., 2010. La nieve en los Picos de Europa: Implicaciones geomorfológicas y ambientales, Cuadernos de Investigación Geográfica, 36 (2): 61-84.

Haeberli, W., 1973. Die Basis-Temperatur der winterlichen Schneedecke als moglicher Indikator für die Verbreitung von Permafrost in den Alpen, Z. Gletscherkd. Glazialgeol., 9: 221-227.

Ishikawa, M., 2003. Thermal regimes at the snow-ground interface and their implications for permafrost investigation. Geomorphology, 52 (1): 105-120. http://dx.doi.org/10.1016/ S0169-555X(02)00251-9

Julián, A. \& Chueca, J., 2007. Permafrost distribution from BTS measurements (Sierra de Telera, Central Pyrenees, Spain): assessing the importance of solar radiation in a mid-elevation shaded mountainous area. Permafrost and Periglacial Processes, 18(2): 137-149. http://dx.doi.org/10.1002/ppp.576

Lugon, R., Delaloye R., Serrano, E., Reynard, E., Lambiel, C. \& González-Trueba, J.J., 2005. Permafrost and Little Ice Age 


\section{2 • A. PISABARRO PÉREZ, E. SERRANO CAÑADAS Y J.J. GONZÁLEZ TRUEBA}

Glaciers Relationships: a Case Study in the Posets Massif, Central Pyrenees, Spain. Permafrost and Periglacial Processes, 15: 207-220. http://dx.doi.org/10.1002/ppp.494

Moscicki, W.J., 2008. Temperature regime on Northern Slopes of Hala Gasienicowa in the Polish Tatra Mountains and its relathionship to permafrost. Studia Geomorphologica Carpatho-Balcanica, 42: 23-40.

Outcalt, S.I., Nelson F.E. \& Hinkel, K.M., 1990. The zero-curtain effect: Heat and mass transfer across an isothermal region in freezing soil. Water Resources Research, 26 (7): 1509-1516. http://dx.doi.org/10.1029/WR026i007p01509

Pellitero, R., 2013. Geomorfología, paleoambiente cuaternario y geodiversidad en el macizo de Fuentes Carrionas-Montaña Palentina. Tesis Doctoral. Departamento de Geografía, Universidad de Valladolid, Valladolid.

Pisabarro, A., 2013. Régimen térmico de suelos y geomorfología de alta montaña en el Macizo Central de Picos de Europa. [On Line] Repositorio trabajos fin de grado Universidad de Valladolid. Disponible en: <http://uvadoc.uva.es:80/handle/10324/7185> [Accedido 23 de Octubre de 2014].

Salvador, F., Salvà, M., Oliva, M. \& Gómez-Ortiz, A., 2012. Régimen térmico del suelo y dinámica periglaciar en el sector de cumbres de Sierra Nevada (planicie del Collado de los Machos, 3.297 m). XII Reunión Nacional de Geomorfología, 631-637, Santander.

Santos González, J., 2011. Glaciarismo y periglaciarismo en el Alto Sil, provincia de León (Cordillera Cantábrica). Tesis Doctoral, Universidad de León, León.
Santos González, J., González Gutiérrez, R.B., Gómez Villar, A. \& Redondo Vega, J.M., 2009. Ground thermal regimes in the vicinity of relict rock glaciers (Cantabrian Mountains, NW Spain). Finisterra, 87: 35-44.

Serrano, E., Martínez de Pisón, E. \& Agudo, C., 2000. El medio periglaciar de alta montaña en el Pirineo Central: aportaciones recientes. En: Peña Monne, J.L., Lozano Tena, M.V., Sánchez Fabre, M. (Eds.): Procesos y formas periglaciares en la alta montaña mediterránea. Instituto de Estudios Turolenses, 45-62, Teruel.

Serrano, E. \& González Trueba, J.J., 2004. Morfodinámica periglaciar en el grupo Peña Vieja (Macizo Central de los Picos de Europa - Cantabria). Cuaternario y Geomorfología, 18 (3-4): 73-88.

Vieira, G.T., Mora, C., Ramos, M., 2003. Ground temperature regimes and geomorphological implications in a Mediterranean mountain (Serra da Estrela, Portugal). Geomorphology, 52: 57-72. http://dx.doi.org/10.1016/S0169-555X(02)00248-9

Von Wakonigg, H., 1996. Unterkühlte Schutthalden. Arbeiten aus dem Institut für Geographie (Graz) 33: 209-223.

Washburn, A.L., 1979. Geocryology. A survey of periglacial processes and environments. Arnold, 406 pp., Londres.

Zhang, T., 2005. Influence of the seasonal snow cover on the ground thermal regime: An overview. Reviews of Geophysics, 43(4): RG4002. http://dx.doi.org/10.1029/2004RG000157 